\title{
Aldosterone-producing mutations in normal adrenal glands
}

New research has identified somatic mutations that lead to increased aldosterone production in aldosteroneproducing cell clusters (APCC), which are found in normal adrenal glands. However, the spectrum of mutations differs from that found in aldosterone-producing adenomas (APAs) and indicates that APCC might also be a distinct source of primary aldosteronism.

Using laser capture microdissection to remove both APCC and normal adrenal gland tissue, the investigators first performed microarray analysis to compare the transcriptomes of the different tissues. Expression of CYP11B2, which encodes aldosterone synthase, was increased by 5.9-fold in APCC compared with cells from the zona glomerulosa $(P=0.0008)$. This increase was also confirmed using quantitative PCR and indicates that APCC are a source of aldosterone.

The team then performed nextgeneration sequencing on paired samples of APCC or control adrenal tissue to identify genetic variants in a panel of genes known to be mutated in APAs. Mutations in CACNA1D and ATP1A1, which code for ion channels or pumps and have previously been reported in APAs, were also found in the APCC. These mutations lead to increased CYP11B2 expression. Notably, no mutations in KCNJ5, which are found in $~ 30 \%$ of APAs, were identified.

Taken together, the authors hypothesize that APCC might be the precursors of APA that have mutations in CYP11B2 and ATP1A1, while APAs that contain KCNJ5 mutations might arise from an alternative pathway.

Tim Geach

Original article Nishimoto, K. et al. Aldosterone-

stimulating somatic gene mutations are common in normal adrenal glands. Proc. Nat/ Acad. Sci. USA doi:10.1073/ pnas. 1505529112 